\title{
Analysis of US Agriculture Market with a New Fama-French Three-Factor Model
}

\author{
Hengjia Zhang ${ }^{1}$, Yanjia Yang ${ }^{2}$, Jiayi Zhu ${ }^{3}$, Liuling Li and Bruce MizrachSi \\ ${ }^{1}$ School of Economics and Management, Tongji University, Shanghai 200092, China; \\ ${ }^{2}$ Economics School, Nankai University, Tianjin 300071, China; \\ ${ }^{3}$ Department of Economics, Yale University, New Haven 208268, US; \\ ${ }^{4}$ Economics School, Nankai University, Tianjin 300071, China; \\ ${ }^{5}$ Economics School Rutgers University, New Jersey, US; \\ azhj_5028@126.com, byanjia_yang@yahoo.com, 'chujiayi1130@126.com, 'liliuling@nankai.edu.c \\ n, ${ }^{\mathrm{e}}$ mizrach@econ.rutgers.edu
}

Keywords: Fama-French 3-factor Model, Standardized Standard Asymmetric Exponential Power Distribution(SSAEPD), EGARCH.

\begin{abstract}
In this article, we propose a new Index for Agriculture Output (AOI) in US. Sample period is from 1960 to 2004. Data is analyzed by a new model, which includes 3 factors in Fama and French (1993), the EGARCH-type volatilities in Nelson(1991) and non-Normal errors in Zhu and Zinde-Walsh(2009). This new agriculture index (AOI) is compared with that constructed by Professor Kenneth R. French. LR, KS and AIC are used for testing parameter restrictions, residual check and model comparison, respectively. MLE is used to estimate parameters via Matlab. Empirical results show the Fama-French 3 factors are still alive! The new model can capture the skewness, fat-tailness and the asymmetric kurtosis in returns, which has better in-sample fit.
\end{abstract}

\section{Introduction}

Treynor(1961), Sharp(1964) and Lintner(1965) propose Capital Asset Pricing Model(CAPM), which is one of the key stones in modern finance. Fama and French(1993) add two more factors such as Size and Book-to-market factors into the CAPM model and create a 3-factor model, which is capable to explain the stock returns better than the CAPM.

After that, a lot of researches about Fama-French 3-factor model have been done. And these researches can be divided into two groups. One group applies this model to different countries and show that this model has powerful explanations. For example, this model can explain the stock markets well for US and other 17 countries in Arshaapalli, Coggin and Doukas(1998), Bangladesh in Baten and Ashraf-UL-Alam(2006), and Korean in Chan(2007).

Others extend this model by finding other factors or changing error assumptions. Carhart(1997) finds the Momentum factor has great effect on stock returns. The Default factor is found by Gharghori, Chan and Faff(2007) and a State Switch variable is added into the model by He(2008). Wang(2012) adds the PE factor. Xiao, Faff and Gharghori(2012) introduce a Sustainability factor. Yin(2011) uses non-Normal error term to propose a new 3-factor model. Yang(2013) adds EGARCH-type volatilities of Nelson (1991) to suggest a new 3-factor model and shows it works better than that of Fama-French(1993).

In the literature of agricultural insurance, different factors that may influence insurance scheme have been studied. Following Rothschild and Stiglitz(1976), in this article, we propose a new index for agriculture output. Then, with this new index, the effect of Fama-French three factors on agriculture market is studied, which is new in the literature. Based on a new 3-factor model in Yang(2013), we try to test following hypotheses: 
1. With EGARCH-type volatilities in Nelson(1991) and non-Normal errors of SSAEPD in Zhu and Zinde-Walsh(2009), are the three factors in Fama and French (1993) still alive in US agriculture market?

2. Can this new 3-factor model beat that of Fama and French(1993).

To answer these questions, the new 3-factor model is estimated by Maximum Likelihood Estimation (MLE). Likelihood Ratio test (LR) is used for parameter restrictions. Kolmogorov-Smirnov test (KS) is used for residual check. And AIC is for model comparison.

Empirical results show with EGARCH-type volatilities and non-Normal errors, the Fama-French 3 factors are still alive in US! EGARCH-type volatility enables the model to respond asymmetrically to positive and negative shocks. The new Fama-French 3-factor model has better in-sample fit than that of Fama-French(1993).

The organization of this article is as follows. The model and methodology are discussed in section . 3. Empirical results and the model comparisons will be presented in section 3 . Section 4 is the conclusions and future extensions.

\section{Model and Methodology}

\subsection{FF-SSAEPD-EGARCH Model}

Considering the EGARCH-type volatility in Nelson (1991) and non-Normal error of SSAEPD in Zhu and Zinde-Walsh(2009), a new 3-factor model is used to analyze agriculture data (denoted as FF-SSAEPD-EGARCH).

$$
R_{t}-R_{f t}=\beta_{0}+\beta_{1}\left(R_{m t}-R_{f t}\right)+\beta_{2} S M B_{t}+\beta_{3} H M L_{t}+u_{t}, t=1,2, \ldots, T,
$$

$u_{t}=\sigma_{t} z_{t}, z_{t} \sim \operatorname{SSAEPD}\left(\alpha, p_{1}, p_{2}\right)$,

$$
\begin{gathered}
\ln \left(\sigma_{t}^{2}\right)=a+\sum_{i=1}^{s} g\left(z_{t-i}\right)+\sum_{j=1}^{m} b_{j} \ln \left(\sigma_{t-j}^{2}\right), \\
g\left(z_{t-i}\right)=\begin{array}{ll}
c_{i} z_{t-i}+d_{i}\left[\left|z_{t-i}\right|-E\left(\left|z_{t-i}\right|\right)\right], \\
\left(c_{i}+d_{i}\right) z_{t-i}-d_{i} E\left(\left|z_{t-i}\right|\right), & \text { if } z_{t-i} \geq 0, \\
\left(c_{i}-d_{i}\right) z_{t-i}-d_{i} E\left(\left|z_{t-i}\right|\right), & \text { else. }
\end{array}
\end{gathered}
$$

Where $\theta=\left(\beta_{0}, \beta_{1}, \beta_{2}, \beta_{3}, \alpha, p_{1}, p_{2}, a,\left\{b_{j}\right\}_{j=1}^{m},\left\{c_{i}\right\}_{i=1}^{s},\left\{d_{i}\right\}_{i=1}^{s}\right)$ are parameters to be estimated. $R_{t}$ is the rate of return for U.S index of agricultural output(AOI) at time $t . R_{f t}$ is the rate of return for the risk-free asset at time $t . R_{m t}$ is the rate of return for the market at time $t . S M B_{t}$ stands for small market capitalization minus big market capitalization. $H M L_{t}$ stands for high book-to-market ratio minus low book-to-market ratio. $T$ is the sample size. $\sigma_{t}$ is the conditional standard deviation, i.e., volatility. The error term $z_{t}$ is distributed as the Standardized Standard Asymmetric Exponential Power Distribution(SSAEPD) proposed in Zhu and Zinde-Walsh(2009).

\section{Empirical Analysis}

\subsection{Data}

In this article, the relationship between US agriculture index and stock returns are analyzed. Yearly data are downloaded from US Department of Agriculture. After calculating the proportion of 
livestock, crop and other outputs, the new Index of Agriculture, $\mathrm{AOI}(\mathrm{Y})$, is created by following formula which is analogous to Industrial Production Index.

Where Qi is the quantity of livestock, crops and others at time $\mathrm{i}$ and W0 is the weight at time 0 . Sample period is from 1960 to 2004.

For comparison, we also download a US monthly agriculture index proposed by Professor Kenneth

R. French from its Data Library. For simplicity, we denote it as AOI_French. Sample period is from 1927:01 to 2011:12.

\subsection{Estimation Results}

For AOI(Y), we find out all coefficients are statistically significant. That means, GARCH terms should be added into 3 factor model and AOI(Y) can earn Alpha returns. Similar results are documented for AOI_French(Y) and AOI_French(M). Hence, we conclude that 3 factors are still alive. For comparison, we also estimate other models. These results show the coefficients in the mean equation are all statistically significant. The values of $\beta 1$ for the new AOI data estimated by this Fama-French 3-factor model are smaller than 1, which indicates that these agriculture data in U.S. are less sensitive to market.

\subsection{Fama-French 3-factor Still Alive}

Likelihood Ratio test (LR) is used to test the joint and individual significance of coefficients in these models. We find out terms are joint statistically significant, which means all data can earn the Alpha returns. Non-Normality is confirmed. ARCH and GARCH terms should be added into Fama-French 3-factor model since they are all statistically significant.

\section{Conclusions and Future Extensions}

In this article, we design a new index for US agriculture output, denoted as AOI(Y). The relationships between US agriculture and stock market are analyzed based on Yang(2013)'s new model. This new model is an extension for Fama and French(1993)'s 3-factor model by introducing a non-Normal error of SSAEPD in Zhu and Zinde-Walsh (2009) and EGARCH-type volatilities in Nelson(1991). For comparison, French's index for US agriculture output (AOI_French(Y) and AOI_French(M)) is analyzed. Likelihood Ratio test (LR) is used for parameter restrictions, Kolmogorov-Smirnov test (KS) for residual check and AIC for model comparison. Maximum Likelihood Estimation method (MLE) is used to estimate models via Matlab.

Empirical results show with EGARCH-type volatilities and non-normal errors, the Fama-French 3 factors are still alive in US! EGARCH-type volatility enables the model to respond asymmetrically to positive and negative shocks. The FF-SSAEPD-EGARCH model fits the data well and has better in-sample fit than others for new AOI data. The Beta coefficients for the AOI $\beta 1$ are all smaller than 1 , which indicates that agriculture market is less sensitive than US stock market.

Future extension will include but not limited to follows. First, other type of volatility such as TGARCH-type volatility or Stochastic Volatility (SV) can be added into the model. Second, different data can be used to check the adequacy of our FF-SSAEPD-EGARCH model. Lastly, we can compare our results with those from other models such as ARIMA and GARCH models.

\section{References}

[1] Andrew R. Bell, Daniel E. Osgood, Benjamin I. Cook, Kevin J. Anchukaities, Geoffrey R. McCarney, Authur M. Greene, Brendan M. Buckley and Edward R. Cook. Paleoclimate Histories Improve Access and Sustainability in Index Insurance Programs. Globe Environmental Change, 2013, 23: 774-781.

[2] Bruce Bjornson and Colin A. Carter. New Evidence on Agricultural Commodity Return Performance under Time-Varying Risk. American Journal of Agricultural Economics, 1997, 8: 918-930. 
[3] Bruce Bjorson and Robert Innes. Risk and Return in Agriculture: Evidence from an Explicit-Factor Arbitrage Pricing Model. Journal of Agricultural and Resource Economics, 1992, 17(2): 232-252.

[4] Bruno Arthur and Ani L. Katchova. Uncertainty and Value Premium: Evidence from the U.S. Working paper, 2013(2).

[5] Cheng Xiucen. The Securitization of Xinjiang Agricultural Disaster Insurance. A Dissertation Submitted for the Degree of Master. 2012, 5. (In Chinese)

[6] Efstratios Loizou, Fotios Chatzitheodoridis, Konstadinos Polymeros, Anastasios Michailidis and Konstadinos Mattas. Sustainable Development of Rural and Coastal Areas: Impacts of A New Fisheries Policy. Land Use Policy, 2014, 38: 41-47.

[7] Fama E F, French K R. The Cross-section of Expected Stock Returns[J]. Journal of Finance, 1992, 47(2): 427-465.

[8] Feng Qingshui and Zhang Xuewei, Development Strategies on Agricultural Insurance under the Building of New Countryside. International Conference on Agricultural Risk and Food Security, 2010, 1: 13-23.

[9] Getaw Tadesse, Bernardina Algieri, Matthias Kalkuhl and Joachim Von Braun. Drivers and Triggers of Iternational Food Price Spikes Volatility. Working Paper, 2013.

[10] Zhou Naijun. The Research of China Agricultural Flood Insurance and Risk Manangement. China Academic Journal, 2013, 151-174. (In Chinese) 